\title{
RAMANUJAN'S FORMULA FOR THE LOGARITHMIC DERIVATIVE OF THE GAMMA FUNCTION
}

\author{
DAVID BRADLEY
}

\begin{abstract}
We prove a remarkable formula of Ramanujan for the logarithmic derivative of the gamma function, which converges more rapidly than classical expansions, and which is stated without proof in the notebooks [5]. The formula has a number of very interesting consequences which we derive, including an elegant hyperbolic summation, Ramanujan's formula for the Riemann zeta function evaluated at the odd positive integers, and new formulae for Euler's constant, $\gamma$.
\end{abstract}

Ramanujan was evidently fond of series expansions and representations of special functions. The unorganized material in the second and third notebooks contain many interesting formulae involving the gamma function, Bessel functions, and hypergeometric functions, to name a few examples. The following remarkable formula for the logarithmic derivative of the gamma function has some very interesting consequences, and should be contrasted with the well-known series representation

$$
\psi(x+1):=\frac{\Gamma^{\prime}}{\Gamma}(x+1)=-\gamma+\sum_{n=1}^{\infty}\left(\frac{1}{n}-\frac{1}{x+n}\right),
$$

$[1$, p.259, 6.3.16].

Theorem. For all $x>0$,

$$
\begin{aligned}
\psi(x+1)= & \frac{\pi}{3} \log x+\frac{1}{2 x}-\frac{1}{4 \pi x^{2}}+\frac{\pi \cot (\pi x)}{e^{2 \pi x}-1}+\frac{\pi \log |2 \sin (\pi x)|}{2 \sinh ^{2}(\pi x)} \\
& +\sum_{k=1}^{\infty} \frac{2 k}{\left(e^{2 \pi k}-1\right)\left(k^{2}-x^{2}\right)}-\frac{\pi}{2} \sum_{k=1}^{\infty} \frac{\log \left|k^{4}-x^{4}\right|}{\sinh ^{2}(\pi k)} \\
& -2 \pi \sum_{k=1}^{\infty} e^{-2 \pi k x}\left(k^{2} \sum_{n=1}^{\infty} \frac{\sin (2 \pi n x)}{k^{2}+n^{2}}-k^{3} \sum_{n=1}^{\infty} \frac{\cos (2 \pi n x)}{n\left(k^{2}+n^{2}\right)}\right) .
\end{aligned}
$$

The theorem is recorded as formula 2, p. 280 in Ramanujan's notebooks [5]. As per usual, Ramanujan gives no proof or explanation. In the sequel, we give a proof, and offer a plausible explanation of how Ramanujan may have arrived at formula (1). But first, we examine the formula more closely, with a view to gaining a better

1991 Mathematics Subject Classification. 33B15, 11Y60.

Presented at the 4th conference of the Canadian Number Theory Association, Halifax, 1994 
understanding of the formula and its many interesting consequences, some of which may not be immediately apparent.

The formula takes some time to absorb, but even an initial glance reveals that the convergence is more rapid than the classical representation. The appearance of the logarithm in a summand is very striking, if not mysterious, and at first may arouse some suspicion, since $\psi(x+1)$ is well-behaved for $x>0$, whereas on the right we have terms which contribute singularities at the positive integers. However, a closer examination reveals that the singularities arising from the various terms actually cancel each other out. For example, from the partial fraction decomposition

$$
\pi \cot (\pi x)=\frac{1}{x}+\sum_{k=1}^{\infty} \frac{2 x}{x^{2}-k^{2}}
$$

it can be seen that the pole contribution from

$$
\frac{\pi \cot (\pi x)}{e^{2 \pi x}-1}
$$

at $x=m \quad\left(m \in \mathbf{Z}^{+}\right)$is cancelled by the $m$ th term in the sum

$$
\sum_{k=1}^{\infty} \frac{2 k}{\left(e^{2 \pi k}-1\right)\left(k^{2}-x^{2}\right)}
$$

leaving only a harmless (finite) quantity. This is a routine exercise. Similarly, using the fact that

$$
|\sin (\pi x)| \sim \pi|x-m| \quad(x \rightarrow m)
$$

one can show that the logarithmic singularity contributed by $\frac{\pi \log |2 \sin (\pi x)|}{2 \sinh ^{2}(\pi x)}$ at $x=m \quad\left(m \in \mathbf{Z}^{+}\right)$is cancelled by the $m$ th term in the sum

$$
\frac{\pi}{2} \sum_{k=1}^{\infty} \frac{\log \left|k^{4}-x^{4}\right|}{\sinh ^{2}(\pi k)}
$$

Carrying out the details of the aforementioned limit calculations provides the following formula for Euler's constant, $\gamma$.

Corollary. Let $m$ be a positive integer. Then

$$
\begin{aligned}
-\gamma+\sum_{j=1}^{m} \frac{1}{j}= & \frac{\pi}{3} \log m+\frac{1}{2 m}-\frac{1}{4 \pi m^{2}}+\sum_{\substack{k=1 \\
k \neq m}}^{\infty} \frac{2 k}{\left(e^{2 \pi k}-1\right)\left(k^{2}-m^{2}\right)} \\
& +\frac{\pi}{2}\left(\frac{\log \pi-\log \left(2 m^{3}\right)-1}{\sinh ^{2}(\pi m)}\right)-\frac{\pi}{2} \sum_{\substack{k=1 \\
k \neq m}}^{\infty} \frac{\log \left|k^{4}-m^{4}\right|}{\sinh ^{2}(\pi k)} \\
& +\frac{1}{2 m\left(e^{2 \pi m}-1\right)}+2 \pi \sum_{k=1}^{\infty} k e^{-2 \pi k m} \sum_{n=1}^{\infty} \frac{k^{2}}{n\left(k^{2}+n^{2}\right)} .
\end{aligned}
$$


Note that for the inner sum in (2) we have [1, p.259, 6.3.17]

$$
\sum_{n=1}^{\infty} \frac{k^{2}}{n\left(k^{2}+n^{2}\right)}=\gamma+\Re \psi(1+i k) .
$$

We have used MAPLE to check (2) with various values of $m$. It is interesting to note that with $m=1$, taking a mere five terms from each of the series indexed by $k$ is sufficient to give $1-\gamma$ accurate to thirteen decimal places: $1-\gamma=0.4227843350984 \ldots$.

Proof of Corollary. Let $x \rightarrow m$ in (1). It suffices to show that

$$
\lim _{x \rightarrow m}\left(\frac{\pi \cot (\pi x)}{e^{2 \pi x}-1}+\frac{2 m}{\left(e^{2 \pi m}-1\right)\left(m^{2}-x^{2}\right)}\right)=\frac{1}{2 m\left(e^{2 \pi m}-1\right)}-\frac{\pi}{2 \sinh ^{2}(\pi m)}
$$

and

$$
\lim _{x \rightarrow m}\left(\frac{\pi \log |2 \sin (\pi x)|}{2 \sinh ^{2}(\pi x)}-\frac{\pi \log \left|m^{4}-x^{4}\right|}{2 \sinh ^{2}(\pi m)}\right)=\frac{\pi\left(\log \pi-\log \left(2 m^{3}\right)\right)}{2 \sinh ^{2}(\pi m)} .
$$

We have

$$
\begin{aligned}
\pi \cot (\pi x) & =\frac{\pi \cos (\pi(x-m))}{\sin (\pi(x-m))}=\frac{\pi\left(1+O(x-m)^{2}\right)}{\pi(x-m)\left(1+O(x-m)^{2}\right)} \\
& =\frac{1}{x-m}+O(x-m), \\
\frac{1}{e^{2 \pi x}-1} & =\frac{1}{e^{2 \pi m}-1}-\frac{2 \pi e^{2 \pi m}}{\left(e^{2 \pi m}-1\right)^{2}}(x-m)+O(x-m)^{2},
\end{aligned}
$$

and

$$
\begin{aligned}
\frac{2 m}{\left(e^{2 \pi m}-1\right)\left(m^{2}-x^{2}\right)} & =\frac{1}{\left(e^{2 \pi m}-1\right)(m-x)} \frac{1}{1+\frac{x-m}{2 m}} \\
& =\frac{1}{\left(e^{2 \pi m}-1\right)(m-x)}\left(1-\frac{x-m}{2 m}+O(x-m)^{2}\right) \\
& =\frac{1}{\left(e^{2 \pi m}-1\right)(m-x)}+\frac{1}{2 m\left(e^{2 \pi m}-1\right)}+O(x-m) .
\end{aligned}
$$

Thus,

$$
\frac{\pi \cot (\pi x)}{e^{2 \pi x}-1}+\frac{2 m}{\left(e^{2 \pi m}-1\right)\left(m^{2}-x^{2}\right)}=\frac{1}{2 m\left(e^{2 \pi m}-1\right)}-\frac{2 \pi e^{2 \pi m}}{\left(e^{2 \pi m}-1\right)^{2}}+O(x-m),
$$

which proves the former. For the latter, we have

$$
\begin{aligned}
L & :=\lim _{x \rightarrow m}\left(\frac{\log |2 \sin (\pi x)|}{\sinh ^{2}(\pi x)}-\frac{\log \left|m^{4}-x^{4}\right|}{\sinh ^{2}(\pi m)}\right) \\
& =\frac{\log 2-\log \left(4 m^{3}\right)}{\sinh ^{2}(\pi m)}+\lim _{x \rightarrow m}\left(\frac{\log |\sin (\pi x)|}{\sinh ^{2}(\pi x)}-\frac{\log |m-x|}{\sinh ^{2}(\pi m)}\right) \\
& =-\frac{\log \left(2 m^{3}\right)}{\sinh ^{2}(\pi m)}+\lim _{x \rightarrow m}\left(\frac{\log \left|\pi(m-x)+O(m-x)^{2}\right|}{\sinh ^{2}(\pi x)}-\frac{\log |m-x|}{\sinh ^{2}(\pi m)}\right) \\
& =\frac{\log \pi-\log \left(2 m^{3}\right)}{\sinh ^{2}(\pi m)}+\lim _{x \rightarrow m}\left(\frac{\log |m-x|+O(m-x)}{\sinh ^{2}(\pi x)}-\frac{\log |m-x|}{\sinh ^{2}(\pi m)}\right) \\
& =\frac{\log \pi-\log \left(2 m^{3}\right)}{\sinh ^{2}(\pi m)}
\end{aligned}
$$


as required.

Instead of letting $x$ tend to a positive integer in (1), one can of course obtain a formula for $\gamma$ by directly substituting $x=m-1 / 2 \quad\left(m \in \mathbf{Z}^{+}\right)$in (1). Jonathan Borwein has used this approach to compute $\gamma$, choosing large $m$ to balance the error terms. However, he reports that Brent's method is computationally superior. Part of the difficulty stems from the need for full precision calculation of the logarithms, and of $e^{\pi k}$. If there is an efficient way of doing these calculations, it is possible that an algorithm based on Ramanujan's formula could beat other methods of computing $\gamma$. [J. Borwein, personal communication.]

Another very interesting, and perhaps unexpected consequence of (1) is obtained by letting $x \rightarrow+\infty$. Since this limiting case is an essential ingredient in the proof of (1), we shall temporarily content ourselves with heuristic reasoning, deferring the rigorous details of the argument to the sequel. As $x \rightarrow+\infty$, most terms drop out, and since $\psi(x+1) \sim \log x$, what remains is

$$
\log x \sim \frac{\pi}{3} \log x-\frac{\pi}{2} \sum_{k=1}^{\infty} \frac{\log \left|k^{4}-x^{4}\right|}{\sinh ^{2}(\pi k)} \quad(x \rightarrow+\infty) .
$$

For large $x$ and small $k, \log \left|k^{4}-x^{4}\right| \sim 4 \log x$, whereas when $\mathrm{k}$ is large, $\sinh ^{2}(\pi k)$ is very large, so that (3) becomes

$$
\log x \sim \frac{\pi}{3} \log x-2 \pi \log x \sum_{k=1}^{\infty} \frac{1}{\sinh ^{2}(\pi k)} \quad(x \rightarrow+\infty) .
$$

For (4) to be true, the coefficients of $\log x$ must agree i.e. we must have

$$
\sum_{k=1}^{\infty} \frac{1}{\sinh ^{2}(\pi k)}=\frac{1}{6}-\frac{1}{2 \pi}
$$

a remarkable result, with an interesting history in and of itself. See [2, Prop. 2.26]. That (5) is a special case of (1) makes Ramanujan's formula all the more intriguing.

An additional consequence of (1) is Ramanujan's remarkable formula for the Riemann zeta function, evaluated at the odd positive integers.

Corollary. Let $N$ be a positive integer. Then

$$
\begin{aligned}
2 N \zeta(2 N+1)+4 N \sum_{k=1}^{\infty} \frac{k^{-2 N-1}}{e^{2 \pi k}-1}+\left(1+(-1)^{N}\right) \pi \sum_{k=1}^{\infty} \frac{k^{-2 N}}{\sinh ^{2}(\pi k)} \\
=(2 \pi)^{2 N+1} \sum_{j=0}^{N+1}(-1)^{j+1}(2 j-1) \frac{B_{2 j}}{(2 j) !} \frac{B_{2 N+2-2 j}}{(2 N+2-2 j) !} .
\end{aligned}
$$

Here, $B_{0}=1, B_{1}=-1 / 2, B_{2}=1 / 6$, etc. are the Bernoulli numbers, defined by

$$
\frac{z}{e^{z}-1}=\sum_{k=1}^{\infty} \frac{B_{k}}{k !} z^{k}, \quad(|z|<2 \pi) .
$$


Since the Bernoulli numbers are all rational, (6) shows that $\zeta(2 N+1)$ is a rational multiple of $\pi^{2 N+1}$ plus either one or two rapidly convergent series, the number depending on the parity of $N$.

Proof of Corollary. The main idea stems from the observation that $\psi(x+1)$ is essentially the ordinary generating function for the sequence $\zeta(2), \zeta(3), \ldots$. More precisely,

$$
\psi(x+1)=-\gamma+\sum_{n=1}^{\infty} x^{n}(-1)^{n+1} \zeta(n+1), \quad(|x|<1) .
$$

Thus, we are tempted to expand both sides of (1) into power series in $x$ and then equate coefficients of $x^{2 N}$. However, since $\log x$ has no Maclaurin series, we first differentiate both sides of (1) with respect to $x$. Since the machinations involved in this differentiation arise in our proof of (1), we shall defer the details to the sequel and content ourselves for the moment with merely reporting that after simplifying the resulting expression, we obtain

$$
\begin{aligned}
\psi^{\prime}(x+1)= & \frac{\pi}{3 x}-\frac{1}{2 x^{2}}+\frac{1}{2 \pi x^{3}}-\frac{\pi^{2} \csc ^{2}(\pi x)}{e^{2 \pi x}-1} \\
& +\sum_{k=1}^{\infty} \frac{4 k x}{\left(e^{2 \pi k}-1\right)\left(k^{2}-x^{2}\right)^{2}}+\sum_{k=1}^{\infty} \frac{2 \pi x^{3}}{\sinh ^{2}(\pi k)\left(k^{4}-x^{4}\right)} .
\end{aligned}
$$

We now expand all terms of (9) into power series in $x$. We have

$$
\begin{aligned}
\sum_{k=1}^{\infty} \frac{4 k x}{\left(e^{2 \pi k}-1\right)\left(k^{2}-x^{2}\right)^{2}} & =\sum_{k=1}^{\infty} \frac{4 x}{k^{3}\left(e^{2 \pi k}-1\right)} \sum_{n=1}^{\infty} n\left(\frac{x}{k}\right)^{2 n-2} \\
& =4 \sum_{n=1}^{\infty} n x^{2 n-1} \sum_{k=1}^{\infty} \frac{k^{-2 n-1}}{e^{2 \pi k}-1} \\
\sum_{k=1}^{\infty} \frac{2 \pi x^{3}}{\sinh ^{2}(\pi k)\left(k^{4}-x^{4}\right)} & =\sum_{k=1}^{\infty} \frac{2 \pi x^{3}}{k^{4} \sinh ^{2}(\pi k)} \sum_{n=0}^{\infty}\left(\frac{x}{k}\right)^{4 n} \\
& =2 \pi \sum_{n=1}^{\infty} x^{4 n-1} \sum_{k=1}^{\infty} \frac{k^{-4 n}}{\sinh ^{2}(\pi k)}
\end{aligned}
$$

and from (8),

$$
\psi^{\prime}(x+1)=\sum_{n=1}^{\infty}(-x)^{n-1} n \zeta(n+1) .
$$

Finally, using (7) and the derivative of the well-known power series for $\pi \cot (\pi x)$, we have

$$
\frac{\pi^{2} \csc ^{2}(\pi x)}{e^{2 \pi x}-1}=\frac{1}{2 \pi x^{3}} \sum_{n=0}^{\infty}(2 \pi x)^{n} \frac{B_{n}}{n !} \sum_{k=0}^{\infty}(-1)^{k+1}(2 k-1) x^{2 k}(2 \pi)^{2 k} \frac{B_{2 k}}{(2 k) !}
$$




$$
\begin{aligned}
= & \frac{1}{2 \pi x^{3}} \sum_{n=0}^{\infty}(2 \pi x)^{2 n} \sum_{j=0}^{n}(-1)^{j+1}(2 j-1) \frac{B_{2 j}}{(2 j) !} \frac{B_{2 n-2 j}}{(2 n-2 j) !} \\
& +\frac{1}{2 \pi x^{3}} \sum_{n=0}^{\infty}(2 \pi x)^{2 n+1} \sum_{j=0}^{n}(-1)^{j+1}(2 j-1) \frac{B_{2 j}}{(2 j) !} \frac{B_{2 n+1-2 j}}{(2 n+1-2 j) !} \\
= & \frac{1}{2 \pi x^{3}}+\frac{\pi}{3 x}+\frac{1}{2 \pi x^{3}} \sum_{n=2}^{\infty}(2 \pi x)^{2 n} \sum_{j=0}^{n}(-1)^{j+1}(2 j-1) \frac{B_{2 j}}{(2 j) !} \frac{B_{2 n-2 j}}{(2 n-2 j) !} \\
& -\frac{1}{2 x^{2}}+\frac{1}{2 \pi x^{3}} \sum_{n=1}^{\infty}(2 \pi x)^{2 n+1}(-1)^{n+1}(2 n-1) \frac{B_{2 n}}{(2 n) !} \frac{B_{1}}{1 !},
\end{aligned}
$$

since $B_{2 n+1-2 j}=0$ for $j=0,1, \ldots, n-1$. Substituting (10) through (13) into (9) yields

$$
\begin{aligned}
\sum_{n=1}^{\infty}(-x)^{n-1} n \zeta(n+1)= & 4 \sum_{n=1}^{\infty} n x^{2 n-1} \sum_{k=1}^{\infty} \frac{k^{-2 n-1}}{e^{2 \pi k}-1}+2 \pi \sum_{n=1}^{\infty} x^{4 n-1} \sum_{k=1}^{\infty} \frac{k^{-4 n}}{\sinh ^{2}(\pi k)} \\
& -\frac{1}{2 \pi x^{3}} \sum_{n=2}^{\infty}(2 \pi x)^{2 n} \sum_{j=0}^{n}(-1)^{j+1}(2 j-1) \frac{B_{2 j}}{(2 j) !} \frac{B_{2 n-2 j}}{(2 n-2 j) !} \\
& -\frac{1}{2 \pi x^{3}} \sum_{n=1}^{\infty}(2 \pi x)^{2 n+1}(-1)^{n+1}(2 n-1) \frac{B_{2 n}}{(2 n) !} \frac{B_{1}}{1 !}
\end{aligned}
$$

The corollary (6) now follows on equating coefficients of $x^{2 N-1}$ in (14).

Remark 1. Equating coefficients of $x^{2 N}$ in (14) gives Euler's celebrated formula $\zeta(2 N)=(-1)^{N}(2 \pi)^{2 N} \frac{B_{2 N}}{(2 N) !} B_{1}=(-1)^{N+1} 2^{2 N-1} \pi^{2 N} \frac{B_{2 N}}{(2 N) !}$, which is also valid when $N=0$, since $\zeta(0)=-1 / 2=B_{1}$. It should also be noted that (6) holds for $N=0$ if we make use of the hyperbolic summation (5) and interpret the term $2 N \zeta(2 N+1)$ as $\lim _{x \rightarrow 0} 2 x \zeta(2 x+1)=1$. In fact, it can be shown that (6) holds for negative integers $N$ as well. For example, the case $N=-1$ in (6) gives

$$
\sum_{k=1}^{\infty} \frac{k}{e^{2 \pi k}-1}=\frac{1}{24}-\frac{1}{8 \pi}
$$

As a further example, let $m>1$ be odd, and let $N=-m$. From (6) and the well-known evaluation $\zeta(1-2 m)=-\frac{B_{2 m}}{2 m}$, we obtain

$$
\sum_{k=1}^{\infty} \frac{k^{2 m-1}}{e^{2 \pi k}-1}=\frac{B_{2 m}}{4 m}
$$

Recalling that $\int_{0}^{\infty} \frac{x^{2 m-1}}{e^{2 \pi x}-1} d x=\frac{B_{2 m}}{4 m}$, we derive a rather striking equality between a sum and an integral in which the summand and the integrand are formally identical:

$$
\sum_{k=1}^{\infty} \frac{k^{2 m-1}}{e^{2 \pi k}-1}=\int_{0}^{\infty} \frac{x^{2 m-1}}{e^{2 \pi x}-1} d x, \quad(\text { odd } m>1)
$$


Remark 2. Let $\alpha, \beta>0$ with $\alpha \beta=\pi^{2}$, and let $N$ be a positive integer. One can derive the slightly more general formula

$$
\begin{aligned}
2 N \alpha^{-N}(\zeta(2 N & \left.+1)+2 \sum_{k=1}^{\infty} \frac{k^{-2 N-1}}{e^{2 \alpha k}-1}\right) \\
& +\alpha^{1-N} \sum_{k=1}^{\infty} \frac{k^{-2 N}}{\sinh ^{2}(\alpha k)}-(-\beta)^{1-N} \sum_{k=1}^{\infty} \frac{k^{-2 N}}{\sinh ^{2}(\beta k)} \\
& =2^{2 N+1} \sum_{j=0}^{N+1}(-1)^{j+1}(2 j-1) \alpha^{N+1-j} \beta^{j} \frac{B_{2 j}}{(2 j) !} \frac{B_{2 N+2-2 j}}{(2 N+2-2 j) !}
\end{aligned}
$$

in the same manner as our proof of (6) by expanding $\frac{\pi \sqrt{\pi \alpha} \csc ^{2}(z \sqrt{\pi \alpha})}{e^{2 z \sqrt{\pi \beta}}-1}$ into partial fractions and then comparing coefficients of like powers of $z$. The author is currently investigating the feasibility of this relatively elementary approach to deriving analogous Ramanujan-like formulae for the Dirichlet $L$-functions via related partial fraction expansions.

Other proofs of (15) have been given via Mellin transforms, the Poisson summation formula, and elliptic modular transformations, to name some examples of various methods employed. See [2] and [3, p. 276] for an extensive bibliography. Since Ramanujan was quite fond of partial fraction expansions, it is possible that our derivation is close to Ramanujan's.

We have seen (2) that specializing $x$ to positive integer values in (1) produces formulae for Euler's constant. However, it should be noted that the following formula for Euler's constant, valid for all real $x>0$, is actually equivalent to (1), namely,

$$
\begin{aligned}
-\gamma= & \frac{\pi}{3} \log x+\frac{1}{4 \pi x^{2}}+\frac{\pi \log |2 \sin (\pi x)|}{2 \sinh ^{2}(\pi x)}-\sum_{k=1}^{\infty} \frac{x^{2}}{k\left(k^{2}+x^{2}\right)} \\
& +\sum_{k=1}^{\infty} \frac{2 k}{\left(k^{2}+x^{2}\right)\left(e^{2 \pi k}-1\right)}-\frac{\pi}{2} \sum_{k=1}^{\infty} \frac{\log \left|k^{4}-x^{4}\right|}{\sinh ^{2}(\pi k)} \\
& -2 \pi \sum_{k=1}^{\infty} e^{-2 \pi k x}\left(k^{2} \sum_{n=1}^{\infty} \frac{\sin (2 \pi n x)}{k^{2}+n^{2}}-k^{3} \sum_{n=1}^{\infty} \frac{\cos (2 \pi n x)}{n\left(k^{2}+n^{2}\right)}\right) .
\end{aligned}
$$

To see the equivalence of (1) and (17), consider the partial fraction expansion of $\frac{\pi \cot (\pi x)}{e^{2 \pi x}-1}$ in the form

$$
\begin{aligned}
\psi(x+1)+\gamma & =\frac{1}{2 x}-\frac{1}{2 \pi x^{2}}+\frac{\pi \cot (\pi x)}{e^{2 \pi x}-1} \\
& +\sum_{k=1}^{\infty} \frac{x^{2}}{k\left(k^{2}+x^{2}\right)}+\sum_{k=1}^{\infty} \frac{4 k x^{2}}{\left(e^{2 \pi k}-1\right)\left(k^{4}-x^{4}\right)}
\end{aligned}
$$

(Entry 8, Chapter 30 [4, p. 374]). Subtracting (18) from (1), we obtain (17). 
If we use the classical formulae $[1, \mathrm{p} .259,6.3 .13,6.3 .17]$

$$
\Im \psi(1+i x)=\frac{\pi}{2} \operatorname{coth}(\pi x)-\frac{1}{2 x} \quad \text { and } \quad \Re \psi(1+i x)=-\gamma+\sum_{k=1}^{\infty} \frac{x^{2}}{k\left(k^{2}+x^{2}\right)}
$$

and substitute the latter in (17), after cancelling $-\gamma$ from both sides there results a formula for $\psi(1+i x)$, valid for $x>0$, analogous to formula (1) for $\psi(1+x)$.

The evaluation (17) was originally proved by Bruce Berndt, Jonathan Borwein, and Will Galway. They showed that the derivatives of both sides of (17) vanish, and that the right side of (17) tends to $-\gamma$ as $x$ tends to infinity. Since (1) and (17) are equivalent, this argument provides a verification of Ramanujan's formula. The question remains as to how Ramanujan arrived at (1) in the first place. We may never know how Ramanujan arrived at some of his results, but in the case of (1), we are able to give a plausible answer to our question.

Ramanujan was in the habit of expanding meromorphic functions into their series of partial fractions, and many such examples can be found in the unorganized pages of the second notebook. However, the following partial fraction expansion (Entry 3 of Chapter 30 [4, p. 359]) has a particular relevance to (1).

$$
\begin{aligned}
\frac{\pi^{2} \csc ^{2}(\pi x)}{e^{2 \pi x}-1}= & \frac{\pi}{3 x}-\frac{1}{2 x^{2}}+\frac{1}{2 \pi x^{3}}-\psi^{\prime}(x+1) \\
& +\sum_{k=1}^{\infty} \frac{4 k x}{\left(e^{2 \pi k}-1\right)\left(x^{2}-k^{2}\right)^{2}}-\sum_{k=1}^{\infty} \frac{2 \pi x^{3}}{\sinh ^{2}(\pi k)\left(x^{4}-k^{4}\right)}
\end{aligned}
$$

Observe that (19) is simply a rearrangement of (9). One is tempted to speculate that Ramanujan, observing the presence of the derivative $\psi^{\prime}(x+1)$, was motivated to integrate both sides of (19) to obtain a formula for $\psi(x+1)$. Carrying out the anti-differentiation reveals a happy correspondence between the terms that arise and several of the terms appearing on the right of (1). To indicate just one example, we see the mysterious $\frac{\pi}{2} \sum_{k=1}^{\infty} \frac{\log \left|k^{4}-x^{4}\right|}{\sinh ^{2}(\pi k)}$ appearing as the anti-derivative of $\sum_{k=1}^{\infty} \frac{2 \pi x^{3}}{\sinh ^{2}(\pi k)\left(x^{4}-k^{4}\right)}$. However, a few terms on the right of (1) remain unaccounted for, of which the double series is the most glaringly obvious. We shall see that the double series arises fairly naturally in the course of proving (1). Accordingly, we now focus on completing this final task.

Proof of Theorem. By the product rule for differentiation, we have

$$
\frac{d}{d x} \frac{\pi \cot (\pi x)}{e^{2 \pi x}-1}=-\frac{\pi^{2} \csc ^{2}(\pi x)}{e^{2 \pi x}-1}-\frac{\pi^{2} \cot (\pi x)}{2 \sinh ^{2}(\pi x)}
$$

and

$$
\frac{d}{d x} \frac{\pi \log |2 \sin (\pi x)|}{2 \sinh ^{2}(\pi x)}=\frac{\pi^{2} \cot (\pi x)}{2 \sinh ^{2}(\pi x)}+\frac{\pi}{2} \log |2 \sin (\pi x)| \frac{d}{d x} \operatorname{csch}^{2}(\pi x) .
$$


Hence, we can rewrite (19) in the form

$$
\begin{aligned}
\psi^{\prime}(x+1)= & \frac{\pi}{3 x}-\frac{1}{2 x^{2}}+\frac{1}{2 \pi x^{3}}+\frac{d}{d x} \frac{\pi \cot (\pi x)}{e^{2 \pi x}-1}+\frac{d}{d x} \frac{\pi \log |2 \sin (\pi x)|}{2 \sinh ^{2}(\pi x)} \\
& +\sum_{k=1}^{\infty} \frac{4 k x}{\left(e^{2 \pi k}-1\right)\left(x^{2}-k^{2}\right)^{2}}-\sum_{k=1}^{\infty} \frac{2 \pi x^{3}}{\sinh ^{2}(\pi k)\left(x^{4}-k^{4}\right)} \\
& -\frac{\pi}{2} \log |2 \sin (\pi x)| \frac{d}{d x} \operatorname{csch}^{2}(\pi x) .
\end{aligned}
$$

But,

$$
\begin{aligned}
-\frac{\pi}{2} \frac{d}{d x} \operatorname{csch}^{2}(\pi x) & =-\frac{d}{d x} \frac{2 \pi e^{2 \pi x}}{\left(e^{2 \pi x}-1\right)^{2}}=\left(\frac{d}{d x}\right)^{2} \frac{1}{e^{2 \pi x}-1}=\left(\frac{d}{d x}\right)^{2} \sum_{k=1}^{\infty} e^{-2 \pi k x} \\
& =\sum_{k=1}^{\infty}(-2 \pi k)^{2} e^{-2 \pi k x}
\end{aligned}
$$

Using this in (20) and integrating both sides with respect to $x$, we obtain

$$
\begin{aligned}
\psi(x+1)= & \frac{\pi}{3} \log x+\frac{1}{2 x}-\frac{1}{4 \pi x^{2}}+\frac{\pi \cot (\pi x)}{e^{2 \pi x}-1}+\frac{\pi \log |2 \sin (\pi x)|}{2 \sinh ^{2}(\pi x)} \\
& +\sum_{k=1}^{\infty} \frac{2 k}{\left(e^{2 \pi k}-1\right)\left(k^{2}-x^{2}\right)}-\frac{\pi}{2} \sum_{k=1}^{\infty} \frac{\log \left|k^{4}-x^{4}\right|}{\sinh ^{2}(\pi k)} \\
& -\int_{x}^{\infty} \log |2 \sin (\pi v)| \sum_{k=1}^{\infty}(-2 \pi k)^{2} e^{-2 \pi k v} d v+C,
\end{aligned}
$$

where $C$ is a constant of integration to be determined. We now let

$$
\begin{aligned}
S(x) & :=\int_{x}^{\infty} \log |2 \sin (\pi v)| \sum_{k=1}^{\infty}(-2 \pi k)^{2} e^{-2 \pi k v} d v \\
& =\int_{0}^{\infty} \log |2 \sin (\pi(x+u))| \sum_{k=1}^{\infty}(-2 \pi k)^{2} e^{-2 \pi k(x+u)} d u .
\end{aligned}
$$

By means of the Laplace transforms

$$
\int_{0}^{\infty} e^{-k t} \sin (n t) d t=\frac{n}{k^{2}+n^{2}} \quad \text { and } \quad \int_{0}^{\infty} e^{-k t} \cos (n t) d t=\frac{k}{k^{2}+n^{2}}
$$

and the well-known Fourier series

$$
\sum_{k=1}^{\infty} \frac{\cos (2 \pi k y)}{k}=-\log |2 \sin (\pi y)|, \quad y \text { real }
$$


we find from (22) that

$$
\begin{aligned}
S(x) & =(2 \pi)^{2} \sum_{k=1}^{\infty} k^{2} e^{-2 \pi k x} \int_{0}^{\infty} e^{-2 \pi k u} \log |2 \sin (\pi(x+u))| d u \\
& =-(2 \pi)^{2} \sum_{k=1}^{\infty} k^{2} e^{-2 \pi k x} \int_{0}^{\infty} e^{-2 \pi k u} \sum_{n=1}^{\infty} \frac{\cos (2 \pi n x+2 \pi n u)}{n} d u \\
& =-2 \pi \sum_{k=1}^{\infty} k^{2} e^{-2 \pi k x} \int_{0}^{\infty} e^{-k t} \sum_{n=1}^{\infty} \frac{\cos (2 \pi n x+n t)}{n} d t \\
& =2 \pi \sum_{k=1}^{\infty} k^{2} e^{-2 \pi k x} \int_{0}^{\infty} e^{-k t} \sum_{n=1}^{\infty} \frac{\sin (2 \pi n x) \sin (n t)-\cos (2 \pi n x) \cos (n t)}{n} d t \\
& =2 \pi \sum_{k=1}^{\infty} k^{2} e^{-2 \pi k x}\left(\sum_{n=1}^{\infty} \frac{\sin (2 \pi n x)}{n} \int_{0}^{\infty} e^{-k t} \sin (n t) d t\right. \\
& =2 \pi \sum_{k=1}^{\infty} e^{-2 \pi n x}\left(k^{2} \sum_{n=1}^{\infty} \frac{\sin (2 \pi n x)}{k^{2}+n^{2}}-k^{3} \sum_{n=1}^{\infty} \frac{\cos (2 \pi n x)}{n\left(k^{2}+n^{2}\right)}\right)
\end{aligned}
$$

where the inversions in the order of summation and integration are justified by absolute convergence. Substituting (23) for the definition of $S(x)$ in (21), we have

$$
\begin{aligned}
\psi(x+1)= & C+\frac{\pi}{3} \log x+\frac{1}{2 x}-\frac{1}{4 \pi x^{2}}+\frac{\pi \cot (\pi x)}{e^{2 \pi x}-1}+\frac{\pi \log |2 \sin (\pi x)|}{2 \sinh ^{2}(\pi x)} \\
& +\sum_{k=1}^{\infty} \frac{2 k}{\left(e^{2 \pi k}-1\right)\left(k^{2}-x^{2}\right)}-\frac{\pi}{2} \sum_{k=1}^{\infty} \frac{\log \left|k^{4}-x^{4}\right|}{\sinh ^{2}(\pi k)} \\
& -2 \pi \sum_{k=1}^{\infty} e^{-2 \pi n x}\left(k^{2} \sum_{n=1}^{\infty} \frac{\sin (2 \pi n x)}{k^{2}+n^{2}}-k^{3} \sum_{n=1}^{\infty} \frac{\cos (2 \pi n x)}{n\left(k^{2}+n^{2}\right)}\right)
\end{aligned}
$$

By letting $x \rightarrow+\infty$ in (24), we can evaluate the integration constant, $C$. In fact, $C$ must equal zero, as a comparison of (24) and (1) reveals. Since most terms in (24) vanish as $x \rightarrow+\infty$, it suffices to show that

$$
\lim _{x \rightarrow \infty}\left(\psi(x+1)-\frac{\pi}{3} \log x+\frac{\pi}{2} \sum_{k=1}^{\infty} \frac{\log \left|x^{4}-k^{4}\right|}{\sinh ^{2}(\pi k)}\right)=0 .
$$

Set $x=N+1 / 2$, where $N$ is a positive integer, and write

$$
\begin{aligned}
\sum_{k=1}^{\infty} \frac{\log \left|x^{4}-k^{4}\right|}{\sinh ^{2}(\pi k)} & =\left(\sum_{k \leq \sqrt{x}}+\sum_{\sqrt{x}<k<x}+\sum_{k>x}\right) \frac{\log \left|x^{4}-k^{4}\right|}{\sinh ^{2}(\pi k)} \\
& =\sum_{k \leq \sqrt{x}} \frac{4 \log x}{\sinh ^{2}(\pi k)}+\sum_{k \leq \sqrt{x}} \frac{\log \left(1-k^{4} / x^{4}\right)}{\sinh ^{2}(\pi k)}
\end{aligned}
$$




$$
\begin{aligned}
& +(4 x \log x) O\left(e^{-2 \pi \sqrt{x}}\right)+O\left(e^{-3 \pi x / 2}\right) \\
= & (4 \log x)\left(\sum_{k=1}^{\infty} \frac{1}{\sinh ^{2}(\pi k)}+O\left(e^{-\pi \sqrt{x}}\right)\right) \\
& +O\left(x^{-2}\right)+O\left(e^{-\pi \sqrt{x}}\right) \\
= & (4 \log x) \sum_{k=1}^{\infty} \frac{1}{\sinh ^{2}(\pi k)}+O\left(x^{-2}\right) .
\end{aligned}
$$

Second, by Stirling's formula,

$$
\psi(x+1)=\log x+O(1 / x), \quad(x \rightarrow+\infty) .
$$

Employing (26) and (27), we see that

$$
\begin{aligned}
\psi(x+1) & -\frac{\pi}{3} \log x+\frac{\pi}{2} \sum_{k=1}^{\infty} \frac{\log \left|x^{4}-k^{4}\right|}{\sinh ^{2}(\pi k)} \\
= & \left(1-\frac{\pi}{3}+2 \pi \sum_{k=1}^{\infty} \frac{1}{\sinh ^{2}(\pi k)}\right) \log x+O(1 / x)
\end{aligned}
$$

as $x$ tends to $+\infty$. However (Berndt [2, Prop. 2.26]),

$$
1-\frac{\pi}{3}+2 \pi \sum_{k=1}^{\infty} \frac{1}{\sinh ^{2}(\pi k)}=1-\frac{\pi}{3}+2 \pi\left(\frac{1}{6}-\frac{1}{2 \pi}\right)=0 .
$$

Using (29) in (28), we find that

$$
\psi(x+1)-\frac{\pi}{3} \log x+\frac{\pi}{2} \sum_{k=1}^{\infty} \frac{\log \left|x^{4}-k^{4}\right|}{\sinh ^{2}(\pi k)}=O(1 / x),
$$

as $x$ tends to $+\infty$. Thus, the limit (25) holds, and the proof of the theorem (1) is complete.

Acknowledgement. The author is grateful to Bruce Berndt, Jonathan Borwein, and Will Galway for their helpful observations.

\section{REFERENCES}

1. M. Abramowitz and I. Stegun, Handbook of Mathematical Functions, Dover, New York, 1972.

2. Bruce C. Berndt, Modular transformations and generalizations of several formulae of Ramanujan, Rocky Mt. J. Math. 7 (1977), 147-189.

3. Bruce C. Berndt, Ramanujan's Notebooks Part II, Springer-Verlag, New York, 1989.

4. Bruce C. Berndt, Ramanujan's Notebooks Part IV, Springer-Verlag, New York, 1994.

5. S. Ramanujan, Notebooks (2 Volumes), Tata Institute of Fundamental Research, Bombay, 1957. 